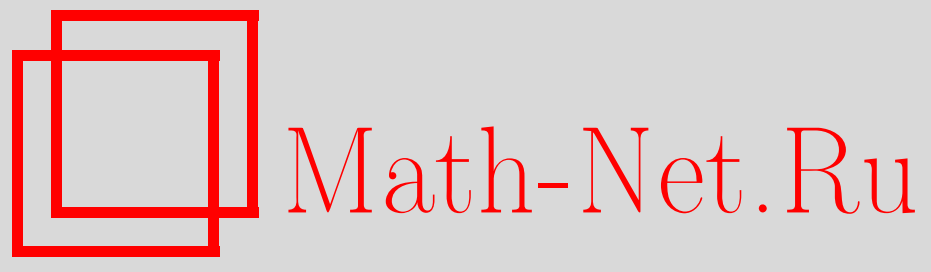

П. В. Ягодовский, Двойственные многозначные группы, УМH, 2009, том 64, выпуск 5, 183-184

DOI: https://doi.org/10.4213/rm9316

Использование Общероссийского математического портала Math-Net.Ru подразумевает, что вы прочитали и согласны с пользовательским соглашением http: //www . mathnet.ru/rus/agreement

Параметры загрузки:

IP: 35.173 .137 .237

26 апреля 2023 г., 10:46:06

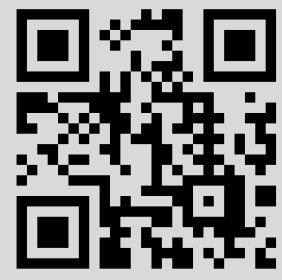




\section{Двойственные многозначные группы}

\section{П. В. Ягодовский}

Цель заметки - построение двойственности в теории многозначных групп. Используя связи многозначных групп с алгебраической комбинаторикой, с теорией обычных групп и их представлений, мы проводим исследование связи построенной двойственности с классическими двойственностями в этих областях - двойственностями Понтрягина и Кавады-Дельсарта. Построенная двойственность является первым шагом в изучении для многозначных групп проблемы, которую (для более общего класса объектов) в работе [1] А. М. Вершик назвал проблемой о дилатации коумножений.

Говорят, что множество $A$ несет на себе структуру $n$-значной групnъ, если задано умножение $\mu: A \times A \rightarrow(A)^{n}$, удовлетворяющее естественным обобщениям аксиом ассоциативной группы. (Подробнее см., например, [2]. Понятие $m$-значной группы было введено В. М. Бухштабером в начале 1990-х годов.) В частности, для каждого элемента $a$ группы должен существовать хотя бы один элемент $b$, обратный ему, т. е. такой, что в произведении $\mu(a, b)$ содержится единица группы. Многозначная группа называется коммутативной, если $\mu(a, b)=\mu(b, a)$ для всех $a, b \in A$. Пусть $C_{a b}^{d}-$ число вхождений элемента $d$ в произведение $\mu(a, b)$. Алгебра $\mathbb{C}(A)$ с выделенным базисом, элементы которого отождествлены с элементами группы $A$, называется груnnовой алгеброй $n$-группы $A$, если ее структурные константы $p_{a b}^{d}$ в выделенном базисе удовлетворяют равенству $p_{a b}^{d}=C_{a b}^{d} / n$. Легко видеть, что групповые алгебры многозначных групп удовлетворяют условию постоянства значности (т. е. $\sum_{k} p_{i j}^{k}=1$ для всех допустимых значений $i$ и $j$ ). Важными примерами многозначных групп являются: 1) косетная группа - многозначная группа на множестве орбит действия на (обычной) группе $G$ группы $H \subset \operatorname{Aut}(G)$ с естественной групповой операцией; 2) группа характеров - многозначная группа на множестве характеров неприводимых представлений данной (обычной) группы $G$ с естественной групповой операцией.

Пусть $P \in \mathrm{GL}(n, \mathbb{C})$ - некоторая матрица, в которой элемент в $i$-й строке на $j$-м месте будет обозначаться через $p_{i}(j)$. Пусть $x_{i}, i=1, \ldots, n$, обозначает $i$-ю строку матрицы $P$. Пусть $\mathrm{A}(P)$ - линейное пространство линейных комбинаций символов $x_{1}, \ldots, x_{n}$ с коэффициентами из $\mathbb{C}$. Для произвольной пары элементов $x_{i}$ и $x_{j}$ рассмотрим Адамарово (покомпонентное) произведение соответствующих им строк, результат которого разложим по базису $x_{1}, \ldots, x_{n}$, состоящему из строк матрицы $P$. Пусть $p_{i j}^{k}$ - коэффициент при $x_{k}$ в этом разложении. Положим $x_{i} \cdot x_{j}=\sum_{k=1}^{n} p_{i j}^{k} x_{k}$. Легко видеть, что эта формула превращает $\mathrm{A}(P)$ в коммутативную алгебру. Определим теперь двойственную по отношению $\kappa \mathrm{A}(P)$ алгебру $\mathrm{A}^{*}(P)$ формулой $\mathrm{A}^{*}(P)=\mathrm{A}\left(P^{t}\right)$.

Рассуждения $\S 2.5$ монографии [3], проведенные там для $C$-алгебр, позволяют установить следующие факты: здесь все неприводимые представления алгебры $\mathrm{A}(P)$ являются одномерными и имеют вид $\Delta_{j}: x_{i} \rightarrow p_{i}(j)$, а сама алгебра $\mathrm{A}(P)$ изоморфна $\mathbb{C} \oplus \cdots \oplus \mathbb{C}$ (здесь $n$ слагаемых). Однако, разные матрицы $P$ одинакового размера приводят к алгебрам, отличающимся своими выделенными базисами.

ПреДложенИЕ. Следующие условия эквивалентны: 1) алгебра $\mathrm{A}(P)$ имеет единицу в числе элементов выделенного базиса; 2) алгебра $\mathrm{A}^{*}(P)$ удовлетворяет условию постоянства значности; 3) одна из строк матрицы $P$ состоит из единиц.

Конечномерная коммутативная алгебра над $\mathbb{C}$ с выделенным базисом $x_{1}, \ldots, x_{n}$ и единицей $x_{1}$ называется nсевдогрупповой, если выполнены условия: 1) для любого $i \in\{1, \ldots, n\}$ существует единственное $i^{\prime} \in\{1, \ldots, n\}$ такое, что: $\left.p_{i i^{\prime}}^{k} \neq 0 ; 2\right)$ антилинейное линейное преобразование алгебры, продолжающее соответствие $x_{i} \rightarrow x_{i^{\prime}}$, является ее автоморфизмом; 3) $\forall i, j, k \in\{1, \ldots, n\} p_{i j}^{k} \in \mathbb{R}$ и $\left.p_{i i^{\prime}}^{1}>0 ; 4\right)$ эта алгебра удовлетворяет условию постоянства значности. Псевдогрупповые алгебры с неотрицательными и рациональными структурными константами являются групповыми 
алгебрами коммутативных конечных многозначных групп, называемых инволютивными. Так, косетные группы и группы характеров являются инволютивными.

Теорема 1. Следующие условия эквивалентны: 1) алгебра $\mathrm{A}(P)$ является псевдогрупповой; 2) алгебра $\mathrm{A}^{*}(P)$ является псевдогрупповой; 3) существуют диагональные матрицы $D_{1}$ и $D_{2}$ с действительными положительными элементами на своих главных диагоналях такие, что матрица $\widehat{P}=D_{1} P D_{2}$ является унитарной, она отличается от матрицы $\widehat{\widehat{P}}$ лишь перестановкой строк и имеет один столбец, и одну строку, состоящие из действительных положительных чисел.

Две коммутативные инволютивные многозначные группы $M$ и $M^{*}$ называются двойственными, если их групповые алгебры двойственны, т. е. если $\mathbb{C}(M) \cong \mathrm{A}(P)$ и $\mathbb{C}\left(M^{*}\right) \cong \mathrm{A}^{*}(P)$. Но не у каждой группы $M$ существует $M^{*}$ : если у алгебры $\mathrm{A}^{*}(P)$ некоторые структурные константы отрицательны или иррациональны, то она не является групповой алгеброй какой-либо многозначной группы.

В алгебраической комбинаторике (см. [3]) доказывается, что для любой $C$-алгебры $\mathcal{C}$ существует матрица $\widetilde{P}$ (называемая собственной) такая, что $\mathcal{C} \cong \mathrm{A}(\widetilde{P})$. В работе [4] введено понятие комбинаторной алгебры - $C$-алгебры специального вида и доказана эквивалентность между категориями групповых алгебр конечных инволютивных многозначных групп и конечномерных комбинаторных алгебр. Из этого соответствия вытекает, что если комбинаторная алгебра $\mathcal{C}(M)$ отвечает многозначной группе $M$ и $\mathcal{C}(M) \cong \mathrm{A}(\widetilde{P})$, то $\mathbb{C}(M) \cong \mathrm{A}(P)$ и $\widetilde{P}=\operatorname{diag}\left(1 / p_{11^{\prime}}^{1}, \ldots, 1 / p_{n n^{\prime}}^{1}\right) \cdot P$. Это соответствие можно продолжить до эквивалентности между категориями псевдогрупповых алгебр и конечномерных коммутативных $C$-алгебр.

Теорема 2. Двойственным псевдогрупповым алгебрам $\mathrm{A}(P)$ и $\mathrm{A}^{*}(P)$ отвечают двойственные (по Каваде-Дельсарту) коммутативные $C$-алгебры $\mathcal{C}(P)$ и $\mathcal{C}^{*}(P)$. Так, двойственным коммутативным инволютивным многозначным группам отвечают двойственные (по Каваде-Дельсарту) коммутативные комбинаторные алгебры.

Примеры. 1. Если $M$ - обычная абелева группа, то существует ее двойственная (в смысле нашего определения) группа $M^{*}$ - это двойственность Понтрягина. 2. Если $M$ - косетная группа абелевой группы $G$ по группе $H \subset \operatorname{Aut} G$, то $M^{*}$ - тоже косетная группа той же группы $G$ по группе $H \subset$ Aut $G$. 3. Если $M$ - косетная группа неабелевой группы $G$ по $G \subset \operatorname{Aut} G$ (имеется в виду, что $G$ действует на себе сопряжениями), то $M^{*}$ будет группой характеров группы $G$. Двойственность из этого примера имеет важное физическое приложение (см. [5]). 4. Если $M$ - косетная группа неабелевой группы $G$ по $H \subset$ Aut $G$, где каждая орбита действия группы $H$ на $G$ представляет собой один класс или объединение нескольких классов сопряженных элементов, то двойственная группа $M^{*}$ существует. 5. Известны примеры ассоциативных схем (см. [3]), у которых часть параметров Крейна иррациональна. Многозначные группы, комбинаторные алгебры которых являются алгебрами смежности таких схем, не имеют двойственных.

Автор выражает глубокую благодарность своему учителю члену-корреспонденту РАН проф. В. М. Бухштаберу за многолетнее внимание и поддержку в работе.

\section{Список литературы}

[1] А. М. Вершик, Функи. анализ и его прил., 41:2 (2007), 24-43. [2] V. M. Buchstaber, Mosc. Math. J., 6:1 (2006), 57-84. [3] Э. Баннаи, Т. Ито, Алгебраическая комбинаторика, Мир, М., 1987. [4] В. М. Бухштабер и др., Функи. анализ и его прил., 30:3 (1996), 12-18. [5] V. M. Buchstaber, M. I. Monastyrsky, J. Phys. A, 36:28 (2003), 7679-7692.

П. В. Ягодовский (Р. V. Yagodovskii)

Московский государственный университет им. М. В. Ломоносова
Представлено В. М. Бухштабером Принято редколлегией 27.08.2009 